\title{
The ups and downs of ageing
}

The many physiological changes that occur as organisms age make it difficult to pinpoint common characteristics of ageing across tissues and species. Two recent studies that have focused on alterations in gene expression during ageing illustrate how headway can be made in this field.

Several expression profiling studies of ageing have been carried out in mice, but the results are difficult to compare as they were produced by different methods and were done on different genetic backgrounds. Zahn and colleagues have tackled this problem by cataloging ageingrelated changes in expression for 16 tissues from a single mouse strain using a standardized methodology. Their results are brought together in a gene expression database named AGEMAP, which is freely accessible.

The AGEMAP study also provides insights into the biology of ageing by highlighting gene sets that have altered expression patterns during ageing in two or more tissues: neural, vascular and steroid-responsive tissues fell into separate categories - the suggestion being that distinct ageing processes occur in these tissue types. Zahn and colleagues also provide evidence that ageing is coordinated among tissues in terms of timing.
Finally, using existing microarray data from worms, flies and humans, the AGEMAP authors found some evidence of conservation in age-related expression changes, for example, for electron-transport-chain genes. However, when the entire sets of genes that show ageing-related changes in expression were compared across mice and humans, the authors found no correlation, indicating that ageing might involve fundamentally different changes in the two species.

Adler and colleagues aimed to identify regulators of the ageing process. They identified a set of transcription-factor binding motifs that are enriched among ageingregulated genes in multiple human and mouse tissues. The binding site for nuclear factor- $\kappa \mathrm{B}(\mathrm{NF}-\kappa \mathrm{B})$ transcription factors (which have known roles in immunity, inflammation and apoptosis) came out as the strongest candidate for involvement in age-dependent gene regulation.

To test whether ageing phenotypes rely on NF- $\mathrm{KB}$ in vivo, Adler and colleagues used transgenic mice that express an inducible inhibitor of NF- $\kappa \mathrm{B}$ activity in the epidermis. Chemically inducing the expression of this transgene in old mice on only one side of the body reduced the expression of more than half of the genes that are upregulated in the skin during ageing to levels that were indistinguishable from those in young mice. Given that the transgene is expressed only in the epidermis, but expression in all skin layers was analysed, these results suggest a role for NF- $\kappa \mathrm{B}$ in the maintenance of expression for many genes that are upregulated in epidermal ageing.

Both studies provide intriguing insights into the biology of ageing, and highlight areas for future research. For example, whereas the AGEMAP study suggests fundamental differences in mouse and human ageing, the findings of Adler and colleagues suggest that the species share regulatory mechanisms. The finding that will perhaps attract most attention is that the ageing programme can be reversed - in mouse epidermis at least - which raises exciting possibilities for counteracting ageingrelated diseases by modifying gene expression.

Louisa Flintoft

ORIGINAL RESEARCH PAPERS Zahn, J. M. et al. AGEMAP: A gene expression database for aging in mice. PLoS Genet. 3, e201 (2007)|Adler, A. S. et al. Motif module map reveals enforcement of aging by continual NF-KB activity. Genes Dev. 30 November 2007 (doi: 10.1101/gad.1588507) WEB SITES

The AGEMAP Gene Expression Database: http://server1-kimlab.stanford.edu/cgi-bin/ index.cgi?Home

Howard Chang's laboratory:

http://changlab.stanford.edu/publication.html 